\title{
Manipulation of Photosynthesis in Grape (Vitis vinifera) CV. 'Flame' by the Application of Two Sucrose Analogs
}

\author{
Tiznado-Hernandez, M.E., J.A. Orozco-Avitia, \\ A.J. Ojeda-Contreras and A.A. Gardea-Bejar \\ ${ }^{1}$ Departamento de Tecnología de Alimentos de Origen Vegetal, \\ Centro de Investigación en Alimentación y Desarrollo, \\ A.C. Carretera a la Victoria km. 0.6 Apartado Postal 1735, Hermosillo, Sonora, 83000, Mexico
}

Received 2013-01-30, Revised 2012-12-19; Accepted 2013-02-09

\begin{abstract}
The translocation of photosynthates from the leaves is important for the accumulation of sugars in grape berries. Two sucrose analogs were applied at post-veraison to determine their effects on leaf photosynthesis. It is hypothesized that the signal of sugar accumulation in leaves will bring a reduction in photosynthesis and an increase in the photosynthate translocation rate to the berries. The experiment was conducted in a commercial Vitis vinifera 'Flame' vineyard. The sucrose analogs used were palatinose and turanose at $5 \mathrm{mM}$. For each treatment, five fully expanded leaves from five different vines were sprayed until run-off. Control leaves were treated with distilled water. Net photosynthesis rate $(\mathrm{Pn})$, stomatal conductance (gs), Photosynthetically Active Radiation (PAR) and leaf temperature (Lt) were measured at 0,10, 20,30,60 and 90 min after the treatment with a LICOR-6200 portable photosynthesis system. Statistical analysis was made by variance analysis based on a randomized complete block design and means separated using the Student-Newman-Keuls test with a confidence level of 0.05. The application of turanose did not affect Pn whereas palatinose reduced the Pn one hour after application. At this point, not statistical differences were found in gs and PAR. It was concluded that the application of palatinose can reduce the net photosynthesis which suggests that it is possible to manipulate photosynthesis by the utilization of sucrose analogs which maybe also increases the translocation of photosynthates from grape leaves to grape berries.
\end{abstract}

Keywords: Grape Leaves, Sucrose Analogs, Palatinose, Turanose, Photosynthesis

\section{INTRODUCTION}

The translocation of photosynthates from the leaf is the phenomena underlying the accumulation of sugars in the berry at post-veraison as shown by the higher activity of phloem at this stage (Greenspan et al., 1996) and by experiments in which the phloem disruption brings about an accumulation of sucrose in leaf (Hunter and Ruffner, 2001). Furthermore, photosynthates translocation from leaf is a light-dependent phenoma tightly coordinated with photosynthesis (Heuvel et al., 2002). Also, it is known that an accumulation of carbohydrate in leaves, reduce the photosynthesis rate, whereas a reduction brings about an increase (Pego et al., 2000; Paul and Foyer, 2001). The models developed to understand the interaction leaf-fruit in Vitis vinifera had shown that the fruit demand directly regulates the leaf photosynthetic activity, in such a way that a high demand increases the photosynthetic activity (Quereix et al., 2001). Furthermore, it is thought that the differences in sugar

\section{Corresponding Author: Tiznado-Hernandez, M.E., Departamento de Tecnología de Alimentos de Origen Vegetal,} Centro de Investigación en Alimentación y Desarrollo, A.C. Carretera a la Victoria km. 0.6 Apartado Postal 1735, Hermosillo, Sonora. 83000, Mexico Tel: 52-(662) 289-24 
concentration giving rise to differences in water potential within the phloem and between the phloem and the fruit regulates the speed of sugar accumulation in the berry (Coombe, 1992; Kuhn et al., 1999). From above, it is feasible to conclude that one way to raise the speed of sugar accumulation in the berry can be done by increasing the amount of sugar in phloem which in turn will increase the concentration difference between the berry and the phloem.

There are very few reports with the objective of increasing the accumulation speed of sugar in berry. It was reported the use of poly carboxylic acids (BasilioHeredia, 1998) and a mixture of gibberelic acid, ascorbic acid and sucrose (Tomer and Kumar, 1977). In both of them, the treatments failed to get an increase in the speed of sugar accumulation in berry. By other side, a treatment with sucrose to tobacco leaf increased the synthesis of organic nitrogen from inorganic one. Furthermore, it was observed a higher biosynthesis of $\alpha$ ketoglutarate which is an important metabolite of the nitrogen assimilation pathways in higher plants (Morcuende et al., 1998). Also, infiltration of sucrose in barley leaf in the dark found post-translational activation of the nitrate reductase enzyme, suggesting that the link between photosynthesis and nitrogen metabolism goes through the presence of carbohydrates (Provan and Lillo, 1999). The induction of an active synthesis of organic nitrogen in these plants by the presence of sucrose in leaf further supports the statement that an active translocation to the sink was taking place since it had been found that the translocation of sucrose to a growing fruit usually goes along with the active synthesis of aminoacids (Paul and Froyer, 2001).

Sucrose is a molecule which can start a signal to inhibit photosyntesis, increase starch synthesis and initiate the active synthesis of proteins involved in sugar transport (Lalonde et al., 1999). By other side, 5 $\mathrm{mM}$ concentrations of the sucrose analogs palatinose and turanose had been shown to trigger the same kind of metabolic responses as sucrose (Fernie et al., 2001). However, they cannot be metabolized by the plant (Loreti et al., 2000) and they cannot be translocated as the normal sucrose (Bornke et al., 2002). Also, the sucrose analogs do not attract insects as the normal sucrose does. Therefore, they can initiate a metabolic signal indicating to the plant that there is a large amount of photosynthates accumulated in the leaf.

Based in the above mentioned, we treat the leaf of Vitis vinifera with solution of sucrose analogs palatinose and turanose with the objective of controlling the net photosynthesis under the hypothesis that the reduction in photosynthesis, which is expected to occur within an hour, as result of a signal indicating large amount of photosynthates in leaf will also induce an active sucrose translocation to the berry.

\section{MATERIALS AND METHODS}

The experiment was conducted in a commercial Vitis vinifera 'Flame' vineyard located $50 \mathrm{~km}$ north of Hermosillo, Mexico.

With the goal to have homogeneous individuals, plants used in the experiment were selected based in the age and diameter of the stem. Also, they also have the same number of bunches. For each treatment, 5 fully expanded and not senescent leaves from 5 different plants were sprayed until dripping using a hand held sprayer. The experiment was conducted between the 9:00 and 11:30 h. The testing of each analog was done in two consecutive days at the same hour in different plants. The sucrose analogs tested were palatinose (6-O- $\alpha$-D-glucopyranosyl-D-fructose) and turanose (3-O- $\beta$-D-glucopyranosyl-D-fructofuranose). It was prepared sucrose analogs solutions of $5 \mathrm{mM}$ including a commercial surfactant. A solution containing only water and the surfactant was used as a control. Leaf temperature (Lt), stomatal conductance (gs), Photosynthesis Rate (PR) and Photosynthetically Active Radiation (PAR) were measured at 0, 10, 20, 30, 60 and 90 min after treatment with a LICOR-6200 portable photosynthesis system (LICOR Environmental Division. Lincoln, Nebraska, U.S.A).

Besides of the determinations mentioned, the leaves were observed for several days after the treatment to look for symptoms of toxic effects like chlorosis or leaf necrosis as a consequence of the treatments with the sucrose analogs.

\subsection{Statistical Analysis}

The statistical analyses were done taking into account the leaf as the experimental unit. The results were studied with variance analysis based on a randomized complete block design, in which the blocks were the different time points. In case of finding statistical differences by variance analysis, means were separated using the Student-Newman-Keuls test with a confidence level of $5 \%$. All statistical analysis was carried out using ver 8.2 of SAS statistical package (SAS Institute, Inc. Cary, N.C. USA). 


\section{RESULTS}

In Fig. 1, it is shown the net photosynthesis, stomatal conductance and the photosynthetically active radiation after $0,30,60$ and 90 min of the experiment initiation for control and palatinose-treated grape leaves. Asterisks are indicating the time points in which there was recorded statistical differences $(p<0.05)$. The values for 10 and $20 \mathrm{~min}$ are not shown in the graph mainly to focus the attention in the period of time in which some changes were observed. It is clear the tendency to have lower net photosynthesis values after $60 \mathrm{~min}$ of the treatment application. By other side, during this time, there was not recorded changes in the stomatal conductances and it is possible to observe that the photosynthetic active radiation was even higher in the case of the palatinose treated leaves.

In Table 1, it is shown the leaf temperature during the experiment for both control and palatinosetreated leaves.

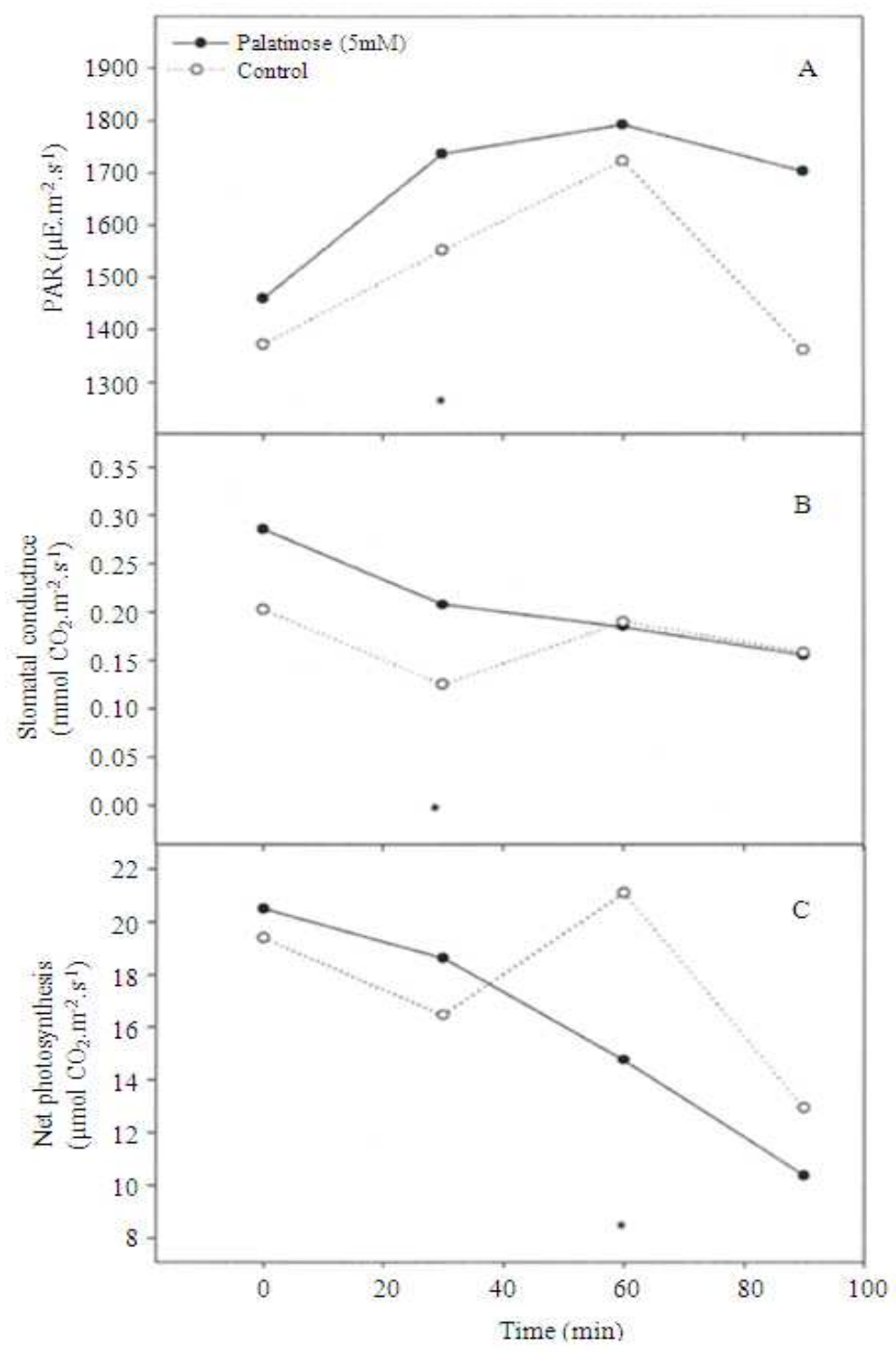

Fig. 1. Effect of the treatment with $5 \mathrm{mM}$ solution of palatinose in grape leaves 
Tiznado-Hernandez, M.E. et al. / American Journal of Agricultural and Biological Sciences 8 (1): 28-33, 2013

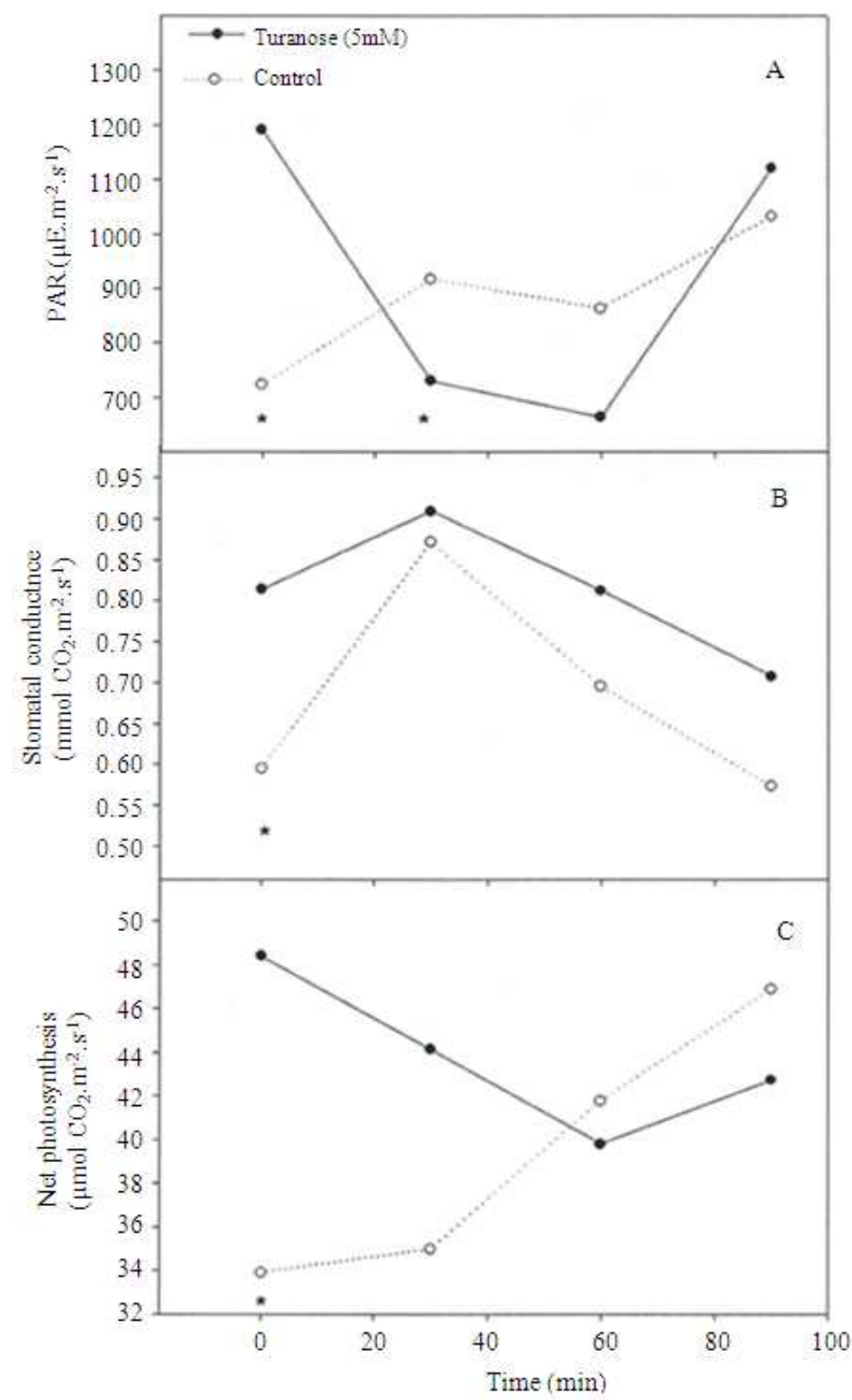

Fig. 2. Effect of the treatment with $5 \mathrm{mM}$ solution of turanose in grape leaves

As it can be seen, higher values $(p<0.05)$ were found for palatinose-treated leaves after 30 and 60 min. Furthermore, after $90 \mathrm{~min}$, these leaves showed lower values $(\mathrm{p}<0.05)$ as compared with the control leaves. It had been found that an increase in temperature of $15^{\circ} \mathrm{C}$ can drastically reduce the net photosynthesis in grape leaves (Ferrini et al., 1995).
In Fig. 2, it is shown the net photosynthesis, stomatal conductance and the photosynthetically active radiation after $0,30,60$ and $90 \mathrm{~min}$ of treatment application for control and turanose-treated grape leaves. The values for 10 and $20 \mathrm{~min}$ are not shown in the graph mainly to focus the attention in the period of time in which some changes were observed. 
Table 1. Temperature of the control and palatinose-treated grape leaves during the period of time in which the measuring of photosynthesis was taking place. Asteriks are indicating the time points in which statistically significant differences were found

\begin{tabular}{llll}
\hline & Treatment & & \\
$\begin{array}{l}\text { Time after } \\
\text { treatment } \\
(\text { min })\end{array}$ & Palatinose & Control & $\begin{array}{l}\text { Statistical } \\
\text { Significance } \\
(\alpha=0.05)\end{array}$ \\
\hline 0 & $37.5 \pm 1.7$ & $37.8 \pm 2.0$ & \\
30 & $40.3 \pm 1.1$ & $38.9 \pm 0.8$ & $*$ \\
60 & $43.5 \pm 1.8$ & $40.6 \pm 1.5$ & $*$ \\
90 & $42.7 \pm 1.1$ & $43.5 \pm 0.8$ & $*$ \\
\hline
\end{tabular}

Table 2. Temperature of the control and turanose-treated grape leaves during the period of time in which the measuring of photosynthesis was taking place. Asteriks are indicating the time points in which statistically significant differences were found

\begin{tabular}{llll}
\hline & Treatment & & \\
& & & \\
$\begin{array}{l}\text { Time after } \\
\text { treatment } \\
(\mathrm{min})\end{array}$ & Turanose & Control & $\begin{array}{l}\text { Statistical } \\
\text { Significance } \\
(\alpha=0.05)\end{array}$ \\
\hline 0 & $37.4 \pm 0.4$ & $35.5 \pm 1.2$ & $*$ \\
30 & $36.2 \pm 1.0$ & $35.9 \pm 1.2$ & \\
60 & $36.7 \pm 1.3$ & $36.2 \pm 1.5$ & \\
90 & $38.9 \pm 1.5$ & $39.6 \pm 0.7$ & \\
\hline
\end{tabular}

As it can be observed, a higher value of net photosynthesis was recorded at the beginning of the experiment for the turanose treated leaves, most likely due to the increased values $(p<0.05)$ in photosynthetic active radiation and stomatal conductance. However, after this time, no statistical differences were observed between turanose treated and control leaves (Fig. 2).

In Table 2, it is shown the leaf temperature during the experiment for both control and turanose-treated leaves. It was only observed higher values $(p<0.05)$ for the turanose treated leaves at the beginning of the experiment. After this time, no differences $(p>0.05)$ were observed in temperature between treated and control grape leaves.

\section{DISCUSSION}

From the results in Fig. 1, it is clear the trend to have a reduction in net photosynthesis $(p<0.05)$ in the palatinose-treated leaves after $60 \mathrm{~min}$ of the application. Also, at this time there was no differences in stomatal conductance and a slight increase in the incident light for the palatinose treated leaves but not statistically significant $(\mathrm{p}>0.05)$. Reduction in both stomatal conductance (Meyer and Genty, 1999) and light (Cartechini and Palliotti, 1995) can inhibit net photosynthesis in higher plants. Further, after $90 \mathrm{~min}$, a lower net photosynthesis was still observed $(p>0.05)$ for palatinose-treated leaves despite the fact that incident light was higher for the control $(p>0.05)$ and no differences in stomatal conductance was observed $(p>0.05)$. In the case of leaf temperature, higher values for the palatinose-treated leaves $(p<0.05)$ were observed at $60 \mathrm{~min}$. However, the real difference was of three degrees which too low in order to attribute to this difference the reduction in net photosynthesis observed. Besides, after $90 \mathrm{~min}$, the palatinose-treated leaves showed lower temperature $(p<0.05)$ than the control and still a lower value of net photosynthesis. Altogether, this data strongly suggest that the reduction on the net photosynthesis observed was due to the treatment, rather than an environmental or physiological status of the leaf. Panel A. Photosynthetically Active Radiation (PAR); Panel B: Stomatal Conductance and Panel C: Net Photosynthesis of control and turanose-treated leaves at different time after the beginning of the treatment. Asterisks are indicating the time points in which statistical differences were found with a confidence of $5 \%$.

In this experiment, no attempt was done to measure photoassimilates translocation, however it is thought than an active translocation was taking place. However, more experimental evidences are needed to further support this last statement.

There was the possibility of observing photobleaching of photosynthetic pigments in the leaves due to the fact that photosynthesis was being reduced by the treatment when there was a high level of light illumination (Velitchkova and Picorel, 2004). However, no apparent damage was observed in the palatinosetreated leaves for up to two weeks after the treatment (data not shown).

In the case of the turanose experiment, there was no statistically significant differences $(p>0.05)$ in net photosynthesis between the control and treated-leaves after 30,60 and 90 min of experiment start. Before experiment initiation, it was observed significant differences in net photosynthesis which correlates with higher values $(p<0.05)$ of photosynthetically active radiation and stomatal conductance (Fig. 2). These findings are important because they strongly support the reliability of the data obtained in this experiment. By other side, in Table 2, it can be observed that higher values $(p<0.05)$ were found for turanose-treated leaves only after the beginning of the experiment. After the initiation of the experiment, no significative differences $(p>0.05)$ were found between control and turanose-treated leaves. Altogether, the data 
obtained from this experiment indicates that the net photosynthesis of leaves was not affected by the sucrose analog turanose.

\section{CONCLUSION}

We had found that palatinose at $5 \mathrm{mM}$ concentration can be used to control within hours the net photosynthesis of grape leaves in vivo when applied topically. Currently, efforts are underway to test the long-term effects of the sucrose analogs on net photosynthesis and the in vivo translocation of photosynthates.

\section{REFERENCES}

Basilio-Heredia, J., 1998. Physiological changes associated with the sugar accumulation on table grapes 'Perlette'. Unpublished Dissertation in Partial Fulfilment of the Requirements for the Degree of Master of Science, Centro de Investigacion en Alimentacion y Desarrollo, A.C. Hermosillo, Sonora, Mexico.

Bornke, F., M. Hajirezaei, D. Heineke, M. Melzer, K. Herbers and U. Sonnewald, 2002. High-level production of the non-cariogenic sucrose isomer palatinose in transgenic tobacco plants strongly impairs development. Planta, 214: 356-364. DOI: 10.1007/s004250100629

Cartechini, A. and A. Palliotti, 1995. Effect of shading on vine morphology and productivity and leaf gas exchange characteristics in grapevines in the field. Am. J. Enol. Vitic., 46: 227-234.

Coombe, B.G., 1992. Research on development and ripening of the grape berry. Am. J. Enol. Vitic., 43: 101-110.

Fernie, A.R., U. Roessner and P. Geigenberger, 2001. The sucrose analog palatinose leads to a stimulation of sucrose degradation and starch synthesis when supplied to discs of growing potato tubers. Plant Physiol., 125: 1967-1977. PMID: 11299376

Ferrini, F., G.B. Mattii and F.P. Nicese, 1995. Effect of temperature on key physiological responses of grapevine leaf. Am. J. Enol. Vitic., 46: 375-379.

Greenspan, M.D., H.R. Schultz and M.A. Mathews, 1996. Field evaluation of water transport in grape berries during water deficits. Physiol. Plantarum, 97: 55-62. DOI: 10.1111/j.1399-3054.1996.tb00478.x

Heuvel, J.E.V., E.D. Leonardos, J.T.A Proctor, K.H. Fisher and J.A. Sullivan, 2002. Translocation and partitioning patterns of ${ }^{14} \mathrm{C}$ photoassimilate from light- and shade-adapted shoots in greenhousegrown 'Chardonnay' grapevines (Vitis vinifera L.). J. Am. Soc. Hortic. Sci., 127: 912-918.
Hunter, J.J. and H.P. Ruffner, 2001. Assimilate transport in grapevines -effect of phloem disruption. Australian J. Grape Wine Res., 7: 118-126. DOI: 10.1111/j.1755-0238.2001.tb00198.x

Kuhn, C., L. Baker, L. Burkle and W.B. Frommer, 1999. Update on sucrose transport in higher plants. J. Exp. Botany, 50: 935-953. DOI: 10.1093/jxb/50.Special_Issue. 935

Lalonde, S., E. Boles, H. Hellmann, L. Baker and J.W. Patrick et al., 1999. The dual function of sugar carriers. Transport Sugar Sens. Plant Cell, 11: 707726. PMID: 10213788

Loreti, E., A. Alpi and P. Perata, 2000. Glucose and disaccharide-sensing mechanisms modulate the expression of alpha-amylase in barley embryos. Plant Physiol., 123: 939-948. PMID: 10889242

Meyer, S. and B. Genty, 1999. Heterogeneous inhibition of photosynthesis over the leaf surface of Rosa rubiginosa L. during water stress and abscisic acid treatment: Induction of a metabolic component by limitation of CO2 diffusion. Planta, 210: 126-131. DOI: $10.1007 / \mathrm{s} 004250050661$

Morcuende, R., A. Krapp, V. Hurry and M. Stitt, 1998. Sucrose-feeding leads to increased rates of nitrate assimilation, increased rates of $\alpha$-oxoglutarate synthesis and increased synthesis of a wide spectrum of amino acids in tobacco leaves. Planta, 206: 394409. DOI: $10.1007 / \mathrm{s} 004250050415$

Paul, M.J. and C.H. Foyer, 2001. Sink regulation of photosynthesis. J. Exp. Botany, 52: 1383-1400. DOI: $10.1093 /$ jexbot $/ 52.360 .1383$

Pego, J.V., A.J. Kortstee, C. Huisjer and S.C.M. Smeekens, 2000. Photosynthesis, sugars and the regulation of gene expression. J. Exp. Botany, 51: 407-416. DOI: 10.1093/jexbot/51.suppl_1.407

Provan, F. and C. Lillo, 1999. Photosynthetic posttranslational activation of nitrate reductase. J. Plant Physiol., 154: 605-609. DOI: 10.1016/S01761617(99)80233-8

Quereix A., R.C. Dewar, J.P. Gaudillere, S. Dayau and C. Valacogne, 2001. Sink feedback regulation of photosynthesis in vines: Measurements and a model. J. Exp. Botany, 52: 2313-2322. DOI: 10.1093/jexbot/52.365.2313

Tomer, N.S. and H. Kumar, 1977. Effect of GA with ascorbic acid and surcrose on perlette grape. Indian J. Hortic., 34: 30-33.

Velitchkova, M.Y. and R. Picorel, 2004. Photobleaching of photosynthetic pigments in spinach thylakoid membranes. Effect of temperature, oxygen and DCMU. Biophys. Chem., 107: 25-32. PMID: 14871598 